\title{
El contexto del deporte en España durante la crisis sanitaria de la COVID-19
}

\author{
The sport context in Spain \\ during the sanitary crisis of COVID-19
}

\author{
David Moscoso-Sánchez \\ Universidad Pablo de Olavide \\ dmoscoso@upo.es \\ ORCID iD: https://orcid.org/0000-0003-4135-1475
}

La crisis epidemiológica del coronavirus ha provocado una parálisis del deporte en España. Ha obligado a quienes practican deporte a permanecer confinados en casa durante dos meses, también ha suspendido los entrenamientos y las pruebas deportivas, las grandes ligas y campeonatos y, en consecuencia, las retransmisiones e informaciones deportivas, paralizando así toda actividad económica ligada al deporte durante ese tiempo.

Con esta contribución se pretende sumar a este debate con un breve diagnóstico sobre el modo como la crisis de la Covid-19 puede haber afectado a la realidad deportiva de nuestro país, a modo de tentativa sobre la actual coyuntura, y cómo debiéramos afrontar el futuro del deporte en el terreno de las ciencias sociales y de las políticas deportivas.

\section{La situación del deporte amateur durante el confinamiento por la crisis sanitaria}

Según la Encuesta de Hábitos Deportivos en España (CSD, 2015), en nuestro país practicaba deporte en los últimos años en torno a un 53\% de la población, es decir, cerca de 25 millones de personas. Desde el punto de vista del deporte amateur y deporte base, durante el mes y medio de confinamiento en el que el Estado de Alarma ha impedido cualquier tipo de prác- tica deportiva en espacios públicos cabe suponer que una parte de esos practicantes se hayan adaptado a la nueva situación, realizando un mayor número de personas la práctica deportiva en su hogar. Al sentir la necesidad de cambiar sus pautas de práctica deportiva, el ciclista habitual en ese tiempo habría continuado realizando su actividad sobre una bicicleta estática, quien acudía frecuentemente al gimnasio para practicar aerobic habría utilizado una aplicación de fitness frente al ordenador y quien antes salía a correr al parque durante este tiempo habría empleado una cinta eléctrica adquirida por Internet.

Con anterioridad al impacto de la pandemia y la declaración de Estado de Alarma, según la misma fuente (MCD, 2015), ya un 19\% de la población realizaba deporte en casa. Entre tanto, el resto lo practicaba al aire libre y en gimnasios privados, instalaciones públicas y centros de trabajo. Aunque no existen datos de mercado publicados, algunos medios de comunicación alertaban durante el mes de marzo que los accesorios deportivos, tales como mancuernas, balones medicinales, barras y discos olímpicos, combas, colchonetas y esterillas, discos deslizantes y material de cross fitness, habían quedado agotados a comienzos del confinamiento en los principales comercios de Internet. Entre tanto, la Encuesta de Hábitos Deportivos en España desprendía en 2015 (MCD, 2015) que el 39\% de la población española disponía enton- 
ces de equipamiento de ejercitación y musculación en sus hogares, una cifra similar a la de la edición de la misma encuesta en 2010 (42\%).

No obstante, cabe presuponer que determinados grupos sociales diferenciados por características sociodemográficas y socioeconómicas —sobre todo, rentas bajas, personas mayores, mujeres y personas con discapacidad - , se habrán encontrado limitados para realizar deporte durante este periodo; algo que no hace más que agrandar la brecha deportiva en España (Moscoso, 2013). Las principales razones que les impedirían la práctica deportiva a estos grupos de población serían, además del impedimento físico de la movilidad por el estado de alarma y el confinamiento, las propias dificultades estructurales, dado que la mayoría de las personas pertenecientes a clases obreras, mayores de 65 años, mujeres y personas con discapacidad, ya venían realizando previamente menos deporte que las personas de clases medias y altas, jóvenes y adultos, hombres y sin discapacidad. Junto a ello, según la misma fuente antes señalada (MCD, 2015), hay que tener en cuenta que entre las rentas bajas el número de personas con aparatos de ejercitación y musculación en casa es diez puntos más bajo entre las clases obreras que entre las clases medias y altas, la personas mayores y con discapacidad realizan casi íntegramente deporte en instalaciones deportivas y al aire libre -las cuales se han encontrado cerradas durante dos meses- y, por último, la mujer aún sigue padeciendo el triple rol femenino (reproductivo, doméstico y laboral), situación que cabe presumir que durante el confinamiento se habrá acusado, al tener en casa durante todo el tiempo a hijos en edad escolar o personas mayores dependientes. En consecuencia, siendo tan relevante la actividad deportiva para la mejora de la salud y el bienestar físico, psíquico y social, la limitación a su acceso no hace más que agrandar la desigualdad social entre los mismos grupos poblacionales.

\section{La situación del deporte de competición y federado}

Igual cambio de comportamiento, o más si cabe, es de presuponer en el caso de los y las deportistas de alto rendimiento y competición. Entre esos millones de deportistas que existen en España, se encuentran también quienes ocupan un lugar destacado en el alto rendimiento. Nos referimos a esa población que desarrolla su actividad deportiva bajo el paraguas de lo que se entiende por "deporte de competición", "federativo", de "alto rendimiento" y/o "profesional". una actividad realizada por los deportistas que dispo- nen de credencial federativa, así como por quienes disfrutan del reconocimiento de alto rendimiento por parte del Consejo Superior de Deportes -máximo órgano del deporte español, perteneciente al Ministerio de Cultura y Deporte del Gobierno de España-, que realizan esta actividad en el marco reglado por los clubes y federaciones deportivas, participando en pruebas y competiciones nacionales e internacionales, se dediquen o no profesionalmente a esta actividad.

Hablamos de un escaso número, apenas unos miles (4.962 deportistas de alto rendimiento, 2.370 futbolistas profesionales, y otros tantos y tantas jugadoras de baloncesto, tenis, balonmano y ciclistas profesionales, de los 3.866.867 deportistas con credencial federativa que existen en nuestro país) (MCD, 2020), pero que ejercen un papel de enorme calado en el ámbito de la representación institucional y la identidad colectiva de federaciones y territorios, puesto que representan a nuestros deportistas, clubes, ciudades y al país en las grandes competiciones. Hablamos de campeones mundiales y medallistas olímpicos, futbolistas, tenistas, ciclistas y deportistas de otras disciplinas, de primera división o división de honor, que en el tiempo de confinamiento y el momento inmediato posterior a la crisis sanitaria provocada por la COVID-19 han sentido truncadas las expectativas de continuidad en sus trayectorias deportivas, e incluso han quedado abocados al paro de la noche a la mañana.

En efecto, las grandes ligas y competiciones nacionales se han interrumpido, tanto las del deporte rey, el fútbol, como las de deportes tradicionales como el baloncesto y balonmano, entre otras muchas. A nivel internacional, pruebas deportivas señeras como la Champions, la Fórmula 1, el Circuito Mundial de Motociclismo, el Tour de Francia o el Giro de Italia, han vivido el mismo designio y, contra todo pronóstico, se han pospuesto los Juegos Olímpicos de Tokio 2020. Ante tal abrumadora realidad, podemos afirmar que el mundo del deporte de competición ha vivido un duro golpe.

Habría que situar en un primer plano a los deportistas que disponen de credencia de Deportistas de Alto Rendimiento, que son aproximadamente 4.962, y que se trata de deportistas que cuentan con una beca del Consejo Superior de Deportes y/o de sus correspondientes federaciones deportivas y patrocinadores, con la que disfrutan de recursos y servicios humanos, alojamiento, técnicos, para su entrenamiento y excelencia en el deporte. Son deportistas que tienen objetivos deportivos a corto, medio y largo plazo, e incluso que forman parte de Equipos Olímpicos para los Juegos Olímpicos de Tokio. 
Durante dos meses han estado entrenando en casa, sufriendo una gran inquietud por su futuro como deportistas, dado que el coste de oportunidad de la carrera deportiva es alto. La situación vivida por parte de estos deportistas es, por lo demás, muy preocupante, porque, con la salvedad de los futbolistas hombres de primera división, todos los demás (especialmente, mujeres deportistas, pero también hombres deportistas, de otras disciplinas deportivas -baloncesto, balonmano, ciclismo, etc. - y de niveles de la competición medios e inferiores) se han encontrado en algunos casos de la noche a la mañana en el paro; algunos de ellos/as teniendo como único ingreso y actividad profesional al deporte. También es grave el caso de quienes, encontrándose en esta situación, no tenían la nacionalidad española, y se han visto obligados a retornar a sus países para mantener el confinamiento junto a sus familias. Especial mención requieren las mujeres deportistas profesionales, dado que su situación es completamente anómala, al no encontrarse en muchos casos sujetas a convenios laborales, por lo que ante esta crisis es previsible que su situación sea la más perjudicada.

\section{La situación de la industria deportiva durante y tras la crisis de la COVID-19}

Con relación al impacto económico para el sector, es evidente imaginárselo si uno piensa en los contratos multimillonarios de publicidad y esponsorización de equipos y competiciones, y que en muchos casos revertirán negativamente en una descapitalización de clubes y federaciones deportivas - para las que tales eventos proveen el grueso de sus ingresos. El sector deportivo representa el 1,5\% del PIB en España, es decir, 15.000 millones de euros anuales (Palco23, 2018).

De todos los pilares de este sector, no cabe duda de que la principal damnificada es la industria de la comunicación, si tenemos presente que buena parte de ese impacto económico lo producen las retransmisiones e informaciones deportivas. Sólo el impacto de las retransmisiones de fútbol en España representa 10.000 millones de euros al año, un 1\% del PIB, teniendo a La Liga como una de las industrias mundiales más poderosas del fútbol televisado, gracias a la calidad del juego de nuestros clubes de fútbol. En términos económicos, La Liga genera, a través de los 42 clubes de fútbol representados en ella, un $1 \%$ del PIB de España (unos 10.000 millones de euros al año), 120.000 puestos de trabajo directos e indirectos, 1.340 millones de euros en impuestos, 2.000 millones de euros al año en derechos audiovisuales (casi la mi- tad fuera de España) a través de 78 contratos en 200 países, siendo la primera industria de exportación de servicios de nuestro país.

Las retransmisiones deportivas han de recordarnos el importante papel que éstas ejercen no sólo en la economía, sino también en el uso del tiempo libre y el ocio. En España, más de 30 millones de personas afirman ver retransmisiones o programas de información deportiva alguna vez o a diario, según el CIS (2015). No existe contenido comunicativo con parangón en la industria de la televisión, la radio o la prensa.

Otros pilares menos visibles en la industria del deporte son las cerca de 35.000 empresas de servicios y productos deportivos, entre las que merece destacar los 5.990 pequeños y medianos comercios de material deportivo que soportan a 225.300 trabajadores y que viven no con menor incertidumbre la crisis del COVID-19 (CSD, 2020). Tampoco merece desdeñarse la importancia del turismo deportivo, que representa un impacto de más de mil millones de euros al año en nuestro país.

- En el caso de los 5.990 pequeños y medianos comercios de material deportivo hemos de tener presente que son establecimientos que se vieron obligados a cerrar al público sus negocios de forma indefinida por el Estado de Alarma. Al ser negocios en su mayoría de carácter tradicional, no están capacitados para mantener sus servicios a través del comercio de Internet. Algo menos de la mitad no cuentan con personal asalariado, es decir, son regentados por el propietario que suele ser autónomo y una cantidad algo mayor tiene menos de 5 asalariados. Por más que hayan podido acogerse a un ERTE o a la ayuda por el cese de actividad, en el caso de los autónomos, los costes de alquiler, luz, impuestos, suponen un gran hándicap, dado que su bolsa de ingresos es muy limitada y, por tanto, la del ahorro para afrontar situaciones como ésta. En el lado opuesto de este sector, están aquellas empresas de venta de material deportivo representadas por grandes compañías como Decatlón y Sprinter, que cuentan con 11.573 y 2.000 empleados respectivamente (Palco, 2020). En su mayoría, en estos casos se han acogido a ERTES y requieren poco apoyo. Aunque es de prever que estas acaben copando mayores cuotas de mercado $y_{\text {, }}$ por tanto, concentrando este sector en el futuro, debido a la presión y desaparición de muchos pequeños negocios familiares de venta de material deportivo al por menor.

- El sector servicios en la industria del deporte se encuentra mayoritariamente representado por las grandes cadenas de gimnasios, así como las empresas sobre las que recae la externalización de servicios en ayuntamientos, diputaciones y otras 
administraciones públicas y empresas privadas y residenciales. De todas esas empresas, que son 28.278, la mayoría no cuentan con asalariados o tienen menos de 5 asalariados. Sin embargo, una minoría de 184 empresas tiene más de 100 asalariados, produciéndose situaciones como la de gimnasios que disponen de más de 2000 empleados cada una (Palco, 2020). Las pequeñas empresas de servicios deportivos pueden encontrarse, al igual que los pequeños comercios de venta al por menor, sujetas a su desaparición, debido a la imposibilidad de asumir los costes de este parón de la actividad económica y deportiva, si no se encuentran ninguna medida de apoyo para paliarlo.

- Por último, en lo que atañe a otro tipo de sectores económicos, como el relativo a la producción de material deportivo, no es fácil conocer sus vicisitudes, pero probablemente sea muy similar a la situación de otros sectores productivos industriales.

\section{Presente y futuro del deporte en España tras la crisis epidemiológica de la COVID-19}

Este contexto no cabe duda que va a incorporar nuevos parámetros morales, culturales y sociales y, con ellos, actitudes, hábitos y comportamientos, que den lugar a una nueva época civilizatoria. Nos encontramos ante una realidad que no es inédita en nuestra historia, sino que más bien es desconcertante para una sociedad que en el último siglo ha vivido un desarrollo del Estado de Bienestar abrumador, convirtiéndose éste en el eje central de todos los pilares de nuestro modelo social. A ello se ha sumado una nueva revolución industrial, la tecnológica, instalando el convencimiento social de nuestra capacidad para vivir siempre con confort, prever cualquier peligro por venir y hacer frente sin dificultad a toda adversidad. De ahí que el alcance de la experiencia de esta crisis epidémiológica haga tambalear algunos de los cimientos de nuestro modelo social.

El deporte no se encuentra ajeno a esta realidad, porque es una actividad social más. No es un compartimento estanco, no es una realidad paralela. Algunos agentes sociales - sobre todo los actores de la industria del deporte-creían que sí. Han comprobado que se equivocaban, porque perdieron de vista la estrecha relación entre esta actividad y la realidad social, algo que siempre hemos defendido desde la sociología del deporte en España (Moscoso, 2006; Sánchez, Moscoso y Piedra, 2020). La crisis epidemiológica de la COVID-19 no ha hecho más que constatar esta afirmación, pues ha provocado una completa parálisis del deporte.
El horizonte por venir sólo puede ser trazado desde la observación de los cambios que ya están produciéndose. Si se aspira a adoptar decisiones en materia deportiva que respondan a la sociedad que está por venir, se deberá atender al principio inspirador de los valores y comportamientos sociales que ya han cambiado. Hete aquí el nudo gordiano que debiera abordarse en cualquier programa de investigación sociológica del deporte en este momento. Entre otras razones, porque cabe pensar que la crisis epidemiológica ha venido para quedarse —al menos, durante los próximos dos años-, no sólo por sus consecuencias inmediatas, sino por las de más largo plazo, concretadas en una crisis económica y una crisis de confianza en los servicios, las instituciones, el consumo, las relaciones sociales, etc.

En este marco, investigar el deporte en la coyuntura actual exige necesariamente contemplar que el nuevo paradigma social gira sobre tres facetas que, más que nunca, marcarán nuestra existencia: la incertidumbre social, la seguridad como nueva cultura y la hipertecnologización de nuestros comportamientos sociales.

Para imaginarse qué será del deporte a partir de esta crisis epidemiológica, tendremos que prever qué cambios se avienen en nuestra sociedad. Dado que el deporte es un reflejo de cómo es una sociedad, las incertidumbres de hoy nos informan de los comportamientos de mañana. La pregunta es si el futuro del deporte será una continuidad de esta nueva realidad, o volveremos a recuperar esa otra versión del deporte que imperaba hasta ahora.

Sea cual sea la respuesta, lo que sí está claro es que necesitaremos un plan de reconstrucción para revitalizar el sector deportivo después de esta importante crisis provocada por el COVID-19. En este sentido, es fundamental impulsar el acceso a la práctica deportiva, apoyar el deporte de competición y federativo y la reactivación económica de empresas y servicios deportivos. Empezando por medidas fiscales que animen la reincorporación a la práctica deportiva entre la población, especialmente entre grupos de rentas bajas para asumir las cuotas de gimnasios e instalaciones deportivas. También se necesitan medidas que pongan en el punto de mira el rescate de los técnicos y deportistas profesionales del deporte de alto rendimiento y de competición en todas sus disciplinas, garantizando la financiación y sostenibilidad del sistema deportivo, y no sólo el del fútbol. Igualmente sería también el momento de impulsar una verdadera política de mecenazgo que atraiga la implicación del sector privado en la financiación del deporte, reduciendo la dependencia sobre las arcas públicas. Y, por último, sería razonable adoptar un 
plan de rescate de la industria del deporte de las empresas de servicios y productos deportivos, en su mayoría autónomos y PYMES, en lugar de priorizar la industria de las retransmisiones deportivas. Todo ello, en aras a la sostenibilidad, equidad social y reactivación económica del sector deportivo en nuestro país.

Sin embargo, las medidas adoptadas hasta el momento por el gobierno de España vaticinan lo contrario, pues tan sólo blindan un modelo de alta industria del deporte y apuntalan el fútbol como 'deporte acaparador'. De nuevo se aplica en España la fórmula de más fútbol, más competición, más espectáculo. Se olvida así que el interés general es el que sitúa también en sus prioridades a los veinticinco millones de personas que, ante la adversidad de la COVID-19, han regresado a las calles, plazas y jardines a formar parte de la base del deporte, que es justo el lugar donde el deporte de competición tiene su fuente. Ojalá miremos al futuro con una mayor visión de lo que supone el deporte de la mayoría social en los procesos de cambio social y su contribución al Estado de Bienestar.

\section{Referencias bibliográficas}

C.D.N. (2020). "Libros, videojuegos y accesorios deportivos: el confinamiento aumenta las ventas online", La Voz de Asturias, 29 de marzo https://www.lavozdeasturias.es/noticia/asturias/2020/03/24/libros-videojuegos-accesorios-deportivos-confinamiento-au- menta-ventas-online/00031585072279682170791. htm

Ministerio de Cultura y Deporte. 2020. "Encuesta de Hábitos Deportivos en España". Acceso 8 de junio http://www.culturaydeporte.gob.es/servicios-al-ciudadano/estadisticas/cultura/mc/deportedata/habitos-deportivos/resultados-habitos-deportivos.html

Ministerio de Cultura y Deporte. 2020. “DEPORTEData". Acceso 8 de junio. http://www.culturaydeporte.gob.es/servicios-al-ciudadano/estadisticas/cultu$\mathrm{ra} / \mathrm{mc} /$ deportedata/portada.html? $\mathrm{L}=0$

Moscoso, David. 2006. "La sociología del deporte en España. Estado de la cuestión". Revista Internacional de Sociología, n. 44:177-204.

Moscoso, David. 2013. "El sueño olímpico y la brecha deportiva". El País, 21 de septiembre. $\quad$ https://elpais.com/elpais/2013/09/16/opinion/1379343283_113729.html

Palco23. 2018. "El deporte en España genera 15.000 millones de euros". Palco23, 7 de junio. https://www.palco23.com/entorno/el-deporte-en-espana-un-negocio-de-15000-millones-de-euros.html

Palc023. 2020. "El deporte asume la avalancha de despidos temporales para combatir el Covid-19". Palco23, 17 de marzo. https://www.palco23.com/ entorno/el-deporte-asume-la-avalancha-de-despidos-temporales-para-combatir-el-covid-19.html

Sánchez, Raúl, Moscoso, David y Piedra, Joaquín. 2020. "The sociology of sport in Spain: Development, current situation, and future challenges". Sport und Gesellschaft, 17(1): 69-95. 